\title{
Modeling rich content for reuse
}

\section{Douglas Coutts}

has consulted to companies in the USA and internationally regarding content and digital asset management. He has been a speaker at conferences internationally, including the annual conference sponsored by ClO Magazine. Doug is a Partner with OTB Solutions Group, a management consulting firm that he co-founded in 2002. Doug's consulting career began with Ernst \& Young, followed by Deloitte Consulting. Prior to becoming a consultant, Doug was VP of Technology at Digital Media Networks in Toronto. He graduated from the Faculty of Engineering, Carleton University, in Ottawa with a Bachelor's degree in Industrial Design.

Keywords: communications, brand consistency, taxonomy, digital asset management, prescriptive reuse, ad hoc reuse

Abstract The growing number and complexity of digital assets is making it increasingly difficult for organizations to locate and retrieve specific assets using traditional DAM systems. Companies that fail to solve this problem risk the erosion of brand consistency and communications with customers, partners and employees. OTB Solutions Group has developed a new approach to digital asset management that provides a flexible solution, which enables users to locate, retrieve and reuse specific assets more efficiently and with more precision.

Technology today enables us to convert our media from analog to digital formats for easier storage, retrieval and editing. Technology also makes it possible for us to transmit those digital files instantly across the room or around the world at the touch of a button. That's the good news.

As organizations convert more and more text, photos, graphics, video and other media into digital formats, however, locating and retrieving specific files has become increasingly difficult. The need to address this challenge has given rise to the field of digital asset management (DAM), and the creation of many different DAM systems, because an organization's digital media files can only truly be called assets if they can be located, retrieved and reused.

Yet the growing complexity of digital assets - such as multiple photos of the same product or nearly identical versions of catalog descriptions in dozens of different languages — is outpacing the ability of traditional DAM systems to evaluate descriptive information, pinpoint specific assets, and identify the best asset for each use. The resulting difficulty not only lowers productivity by causing people to spend more time unsuccessfully searching for the digital assets they need, it also threatens to erode brand consistency and to undermine effective communication with target audiences.

A global corporation that was facing these challenges recently hired OTB Solutions Group to develop a new approach to DAM that would improve its ability to reuse digital assets and enhance its communication with customers, distributors and employees 
worldwide. Even though we're still working on this project, it provides a good case study for a more efficient, new approach to digital asset management.

\section{A CURRENT CASE STUDY}

Our client is a Fortune 250

manufacturing company, which sells its products to millions of customers through thousands of local distributors in 80 countries. The company has more than 400 print publications and over 50,000 web pages for targeted communication with customers and distributors worldwide. Almost all of that content is localized for the many different languages, cultures and local regulations of the countries where it appears.

For this engagement, our client had several objectives:

1. to increase the consistency of brand messages across all communication channels, primarily print and web publications;

2. to reduce the cost of communications by rationalizing the company's portfolio of publications to identify and reduce redundancy, and by increasing the reuse of assets across all markets and communication channels;

3. to reduce the time and labor needed to recreate or reuse assets;

4. to streamline the process of localizing, reviewing and approving assets; and

5. to move toward a more automated process for producing publications.

The solution we are developing and implementing for our client is on track to achieve all of these objectives. In addition, the new solution is expected to pay for itself very quickly, and to return cost savings of more than $\$ 7 \mathrm{~m}$ annually.

\section{THE PROBLEM}

The first problem we faced was simply the number and complexity of digital assets our client owned and needed to reuse, to communicate their messages and reinforce their brand worldwide. Their content includes simple assets such as images, and compound assets such as web pages and print publications. While some of their publications and web pages are one-offs, new editions of most are published monthly, quarterly or annually.

Currently, our client's existing DAM system has about 1,500 users globally. Broadly speaking, these users fall into two categories, determined by the type of assets they're searching for and how they intend to reuse those assets.

First, there are the users who want to find out what assets of a certain type are available. For example, users in this category may be wondering, "What images do we have that show customers using our products?" These users are interested in what we call "ad hoc" reuse. Secondly, there are users who need to locate a particular asset for a specific purpose. For example, users in this category would be asking, "What is the approved master product description and image for use in our localized print and web catalogs?" These users are interested in "prescriptive" reuse.

A common complaint about our client's existing DAM system is that users can't easily find the assets they need. Although the system includes a sophisticated search capability that leverages a clearly defined classification taxonomy, searches typically result in 
either no matches or several dozen matches. Users can browse through the hierarchy, of course, but it's a slow process. In addition, once users locate an asset using this system, they usually need to view it to verify that they really have the specific asset they need. If the asset isn't correct, then they must either search again or look at the other assets found by their original query to see whether the one they need is on that list.

When we analyzed this more closely we found that search results often included many logical duplicates. Sure, they were all separate asset files, but those files were just different renditions of the same content. For example when users searched for a specific product image, the system typically would return several red, green and blue (RGB) assets plus more than a dozen color corrected cyan, magenta, yellow and black (CMYK) assets. Using this system, a query that found ten products often would return a list of more than 100 assets.

Such search results weren't very helpful, but neither were they incorrect, because each asset file returned matched exactly the search query that had been entered. It's just that our client's existing DAM system was unable to request, recognize or distinguish between the individual characteristics and subtle variations that made each image unique. Clearly, we needed to find a way to correct that shortcoming.

A traditional solution adopted by many companies is simply to add more metadata to each asset to support filtering searches, but our client found that the costs and compliance issues associated with such an approach would be unacceptable. In our search for new approaches to solve this problem we found some that may be broadly useful.

\section{THE SOLUTION OVERVIEW}

The most critical feature of the solution we developed for our client is that it helps users quickly find the specific assets they need to support both ad hoc and prescriptive reuse.

Our client had already tried traditional approaches of classifying simple and compound assets in a multifaceted taxonomy, but with only partial success. This certainly presented an opportunity to improve and refine the taxonomy and the DAM system that had already been implemented, but we believed what our client really needed was a whole new approach.

Our solution not only builds upon existing taxonomic approaches, but also borrows from basic communications theory and system modeling approaches. We believe this new approach not only solves our client's problem, but also applies broadly to DAM problems faced by many other companies that must store, retrieve and reuse a growing number of increasingly complex digital assets.

Our approach is based on three core strategies to support search. First, we reduced the size of the universe of assets that had to be searched for each query, providing users with the means to search across a smaller set of objects. We accomplished this by defining "abstract content objects" that each correspond to several assets. The types of content objects in our rich content model include:

- Messages - a collection of content objects that are used for a specific communications context, such as a product launch message, to one or more 
target audiences through one or more communications channels.

- Content elements - a collection of assets that all have the same meaning, such as the RGB and CMYK versions of the same image.

- Content classes - structured content that can be represented in a normalized data structure, which might include categories such as product, distributor, customer, etc.

- Attributes - metadata that specifically characterize or classify a content element, message or content class.

- Asset renditions - the different assets that are renditions of one content element instance, such as two resolutions of the same image, a low-resolution version for the web and a high-resolution version for print.

The relationship among these content objects is depicted in Figure 1. The hierarchy of abstract content objects provides multiple levels of granularity for search. Coarser grained objects (ie message type) represent a larger collection of assets than fine grained objects (ie content element). However, this is different from simply moving up and down with a taxonomic hierarchy. Content objects exist notionally outside of the taxonomic hierarchy: they are a user-centric reflection of the work of communications professionals and they possess the key characteristic of uniqueness.

We defined what made content objects unique, by identifying the metadata attributes that would uniquely specify instances for each type of content object. This was an essential step in enabling the system to support prescriptive reuse. The purpose was not to impose a specific notion of uniqueness, but rather to create a flexible framework that would allow people to define uniqueness differently for each level of granularity.

\section{Solution Overview}
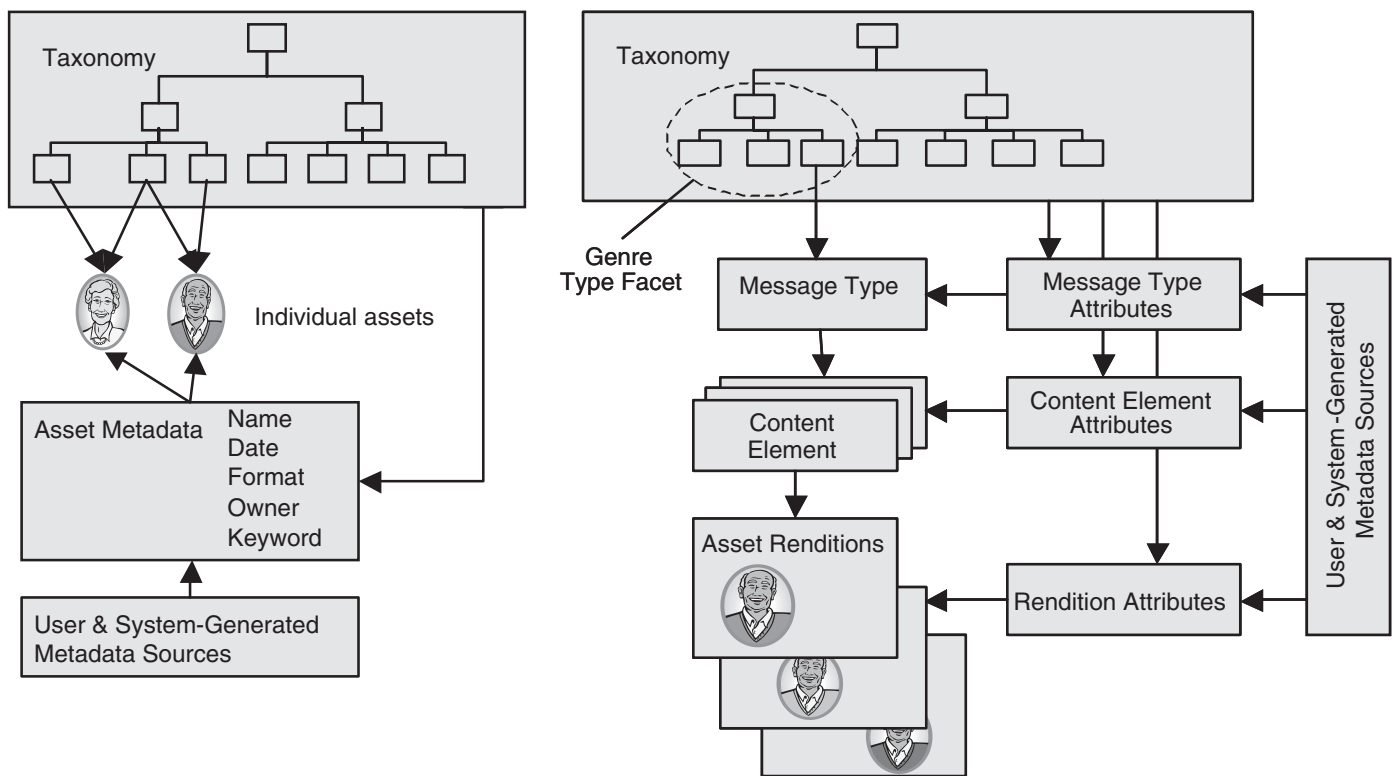

Figure 1: Current versus proposed content object model 


\section{Content Classes}

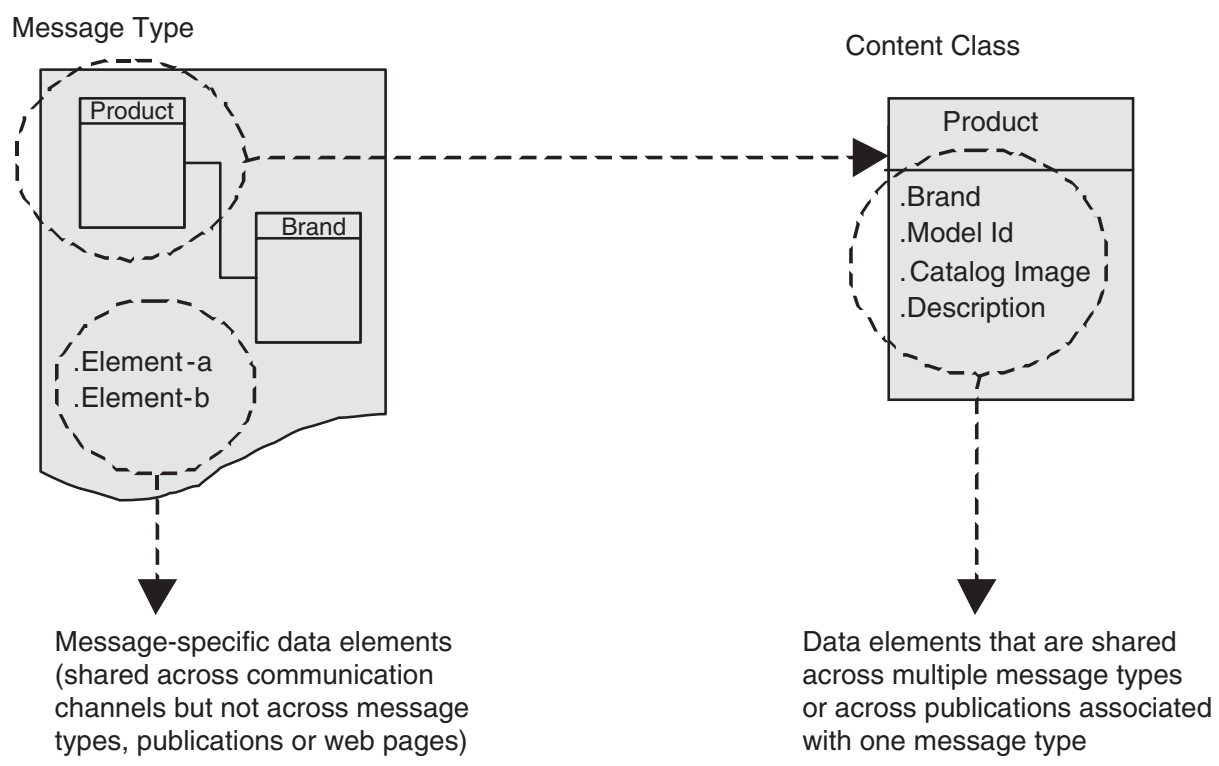

Figure 2: View of the content elements and classes for a message type

Finally, we aligned the definition of content classes and their attributes with enterprise information systems, to enable the system to populate searchable metadata fields automatically. Many of the content elements we identified corresponded to data that are stored and managed in enterprise information systems, which manage a lot of data that are useful in a search context.

To accomplish the goal of aligning our rich content model with enterprise information systems, we applied Universal Modeling Language (UML) modeling. A "content class" in our model has essentially the same meaning as the data structure that comprises a UML Class. Unlike the UML data structure, however, our model distinguishes between two types of data elements:

- Content elements - an atomic unit of content

- Attributes - metadata that classifies the content class
In some cases, content elements are also attributes (see Figure 1).

\section{THE PROCESS}

We started the process of developing this solution by conducting a bottom-up analysis of more than 400 publications and sampling hundreds of web pages and dozens of multimedia artifacts to create a publication inventory and to identify reusable content objects. We then analyzed the frequency with which these artifacts appeared in publications to identify the content objects that had the highest reuse value.

With that accomplished, we analyzed the publication inventory and the initial content object inventory to identify message types and content elements. Examples of the message types and content elements discovered through this analysis are depicted in Tables 1 and 2. We defined taxonomy facets for messages, message audience, and communication context along with 
Table 1: Examples of message type objects

\begin{tabular}{|c|c|c|c|c|c|}
\hline Audience & Context & Message Type & Web & Print & $?$ \\
\hline \multirow[t]{6}{*}{ Customer } & \multirow[t]{2}{*}{ Learn } & Customer Testimonial & $\checkmark$ & $\checkmark$ & \multirow[t]{13}{*}{$\checkmark$} \\
\hline & & Product Description & $\checkmark$ & $\checkmark$ & \\
\hline & Compare & Product Selection Guide & & $\checkmark$ & \\
\hline & Buy & Catalog Item & $\checkmark$ & & \\
\hline & Support & User Manual & $\checkmark$ & $\checkmark$ & \\
\hline & & Tips \& Techniques & & $\checkmark$ & \\
\hline \multirow[t]{7}{*}{ Reseller } & Learn & & & & \\
\hline & Compare & Competitive Positioning & $\checkmark$ & & \\
\hline & & Bulletin & & & \\
\hline & Buy & Brand Description/ & $\checkmark$ & $\checkmark$ & \\
\hline & & Positioning Message & & & \\
\hline & & Reseller Incentive Message & & & \\
\hline & Support & Service Manual & $\checkmark$ & & \\
\hline
\end{tabular}

Table 2: Examples of content elements identified through analysis of current publications

\begin{tabular}{|c|c|}
\hline Message Type & Content Element \\
\hline \multirow[t]{5}{*}{ Product Description } & Product Name \\
\hline & Catalog Image \\
\hline & Catalog Description \\
\hline & Long-form Description \\
\hline & Product Specifications \\
\hline \multirow[t]{6}{*}{ Catalog Item } & Order Code \\
\hline & Product Name \\
\hline & Package Name \\
\hline & Quantify \\
\hline & Price \\
\hline & Package Image \\
\hline \multirow[t]{5}{*}{ Customer Testimonial } & Customer Name \\
\hline & Testimonial Image \\
\hline & Product \\
\hline & Brand \\
\hline & Testimonial Story \\
\hline
\end{tabular}

traditional facets for location, organization structure, brand hierarchy etc as shown in Table 3. Through interviews with users and user-centered analyses of content management processes, including content creation, management, assembly and publishing, we also identified additional metadata used for searches. We then integrated these metadata with the taxonomy to identify attributes of content message types and content elements.

At this stage, we reviewed enterprise information system models so that we could align the naming conventions and identify additional attributes of content objects. We then "normalized" the

Table 3: Aligning the enterprise taxonomy with the rich content model by identifying facets that combine to uniquely identify message type objects

\begin{tabular}{ll}
\hline Facet & Definition \\
\hline $\begin{array}{l}\text { Message Genre } \\
\text { Audience }\end{array}$ & $\begin{array}{l}\text { A hierarchy of content genre with message types at the leaf level. } \\
\text { List of target audience types for messages. } \\
\text { Employees, contractors, vendors, etc. }\end{array}$ \\
Communication Context & $\begin{array}{l}\text { The context of communicating with a specific audience. } \\
\text { (eg for Customer - learn, compare, negotiate, buy, support) }\end{array}$ \\
Business Functions \& Processes & $\begin{array}{l}\text { Business functions, processes, and techniques } \\
\text { (regardless of organizational structure). }\end{array}$ \\
Organizational Unit & $\begin{array}{l}\text { Organizational structure. Names of departments, etc. } \\
\text { Business topics relevant to your business. }\end{array}$ \\
Geographic Markets & $\begin{array}{l}\text { Hierarchy of geographic regions by which we manage our business } \\
\text { Customer market segments. } \\
\text { Brand Hierarchy } \\
\text { Products \& Services }\end{array}$ \\
\hline
\end{tabular}


Defining Uniqueness
Identifying the attributes that uniquely identify instances of each content object enables the system to support prescriptive reuse.

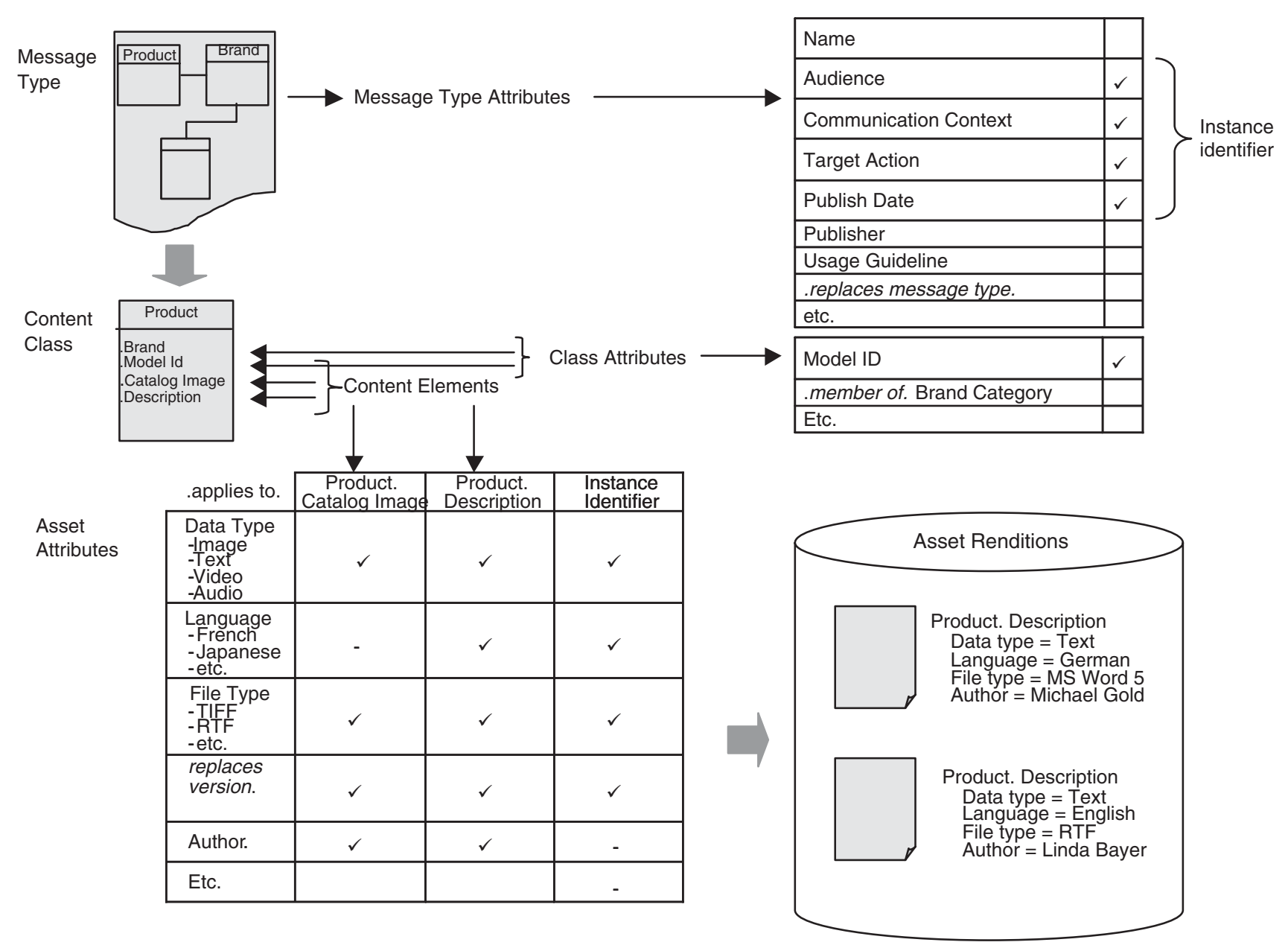

Figure 3: Defining uniqueness for each type of content object

content model to associate each content element and attribute with a single content class. An example of how the class model maps onto message types is shown in Figure 3. Working with the business stakeholders, we identified the attributes that would uniquely define each message type, content element, content class and asset rendition. The next result of this work is a content model with the type of detail depicted in Figure 4.

We are currently in progress testing and refining our rich content model, using sample search scenarios designed to challenge its completeness, correctness and usability. The test results to date have validated the basic approach. Our next step is to design and implement functionality in the new asset management system that enables content creators, consumers and digital libraries to work with the content objects identified in our model.

\section{CONCLUSION}

The new approach to organizing and finding assets that my colleagues and I 
have developed builds on well proven taxonomic approaches, but it goes much further. Our approach shifts the focus from managing digital assets to managing digital objects. It provides multiple levels of granularity as a starting point for searching, by defining different types of content objects and associated metadata. It supports prescriptive reuse of digital assets through content object instance identifiers. In addition, it enables a guided search, within search functionality, to help users zero in on the required content without sifting through large numbers of search results.

To sum up, our approach provides a flexible DAM solution that enables users to locate, retrieve and reuse digital assets more quickly and efficiently, and with much more precision. The potential benefits for organizations adopting this approach are lower costs, higher productivity, significantly reduced brand dissonance, and more consistent communications with target audiences across all channels. 